This is the final peer-reviewed accepted manuscript of:

S. Sindaco, S. Nanni, C. Aguzzi, L. Roffia and T. Salmon Cinotti, "Enabling Context Aware Tuning of Low Power Sensors for Smart Agriculture," 2020 IEEE International Workshop on Metrology for Agriculture and Forestry (MetroAgriFor), Trento, 2020, pp. 114-118, doi: 10.1109/MetroAgriFor50201.2020.9277635.

The final published version is available online at: https://doi.org/10.1109/MetroAgriFor50201.2020.9277635

Rights / License:

The terms and conditions for the reuse of this version of the manuscript are specified in the publishing policy. For all terms of use and more information see the publisher's website.

This item was downloaded from IRIS Università di Bologna (https://cris.unibo.it/)

When citing, please refer to the published version. 


\section{Enabling Context Aware Tuning of Low Power Sensors for Smart Agriculture}

\author{
$1^{\text {st }}$ Simone Sindaco \\ Advanced Research Center \\ On Electronic Systems \\ "Ercole De Castro" (ARCES) \\ University of Bologna \\ Bologna, Italy \\ simone.sindaco2@unibo.it
}

\author{
$2^{\text {nd }}$ Stefania Nanni \\ Lepida ScpA \\ Bologna, Italy \\ stefania.nanni@lepida.it
}

\author{
$3^{\text {rd }}$ Cristiano Aguzzi \\ Advanced Research Center \\ On Electronic Systems \\ "Ercole De Castro" (ARCES) \\ University of Bologna \\ Bologna, Italy \\ cristiano.aguzzi@unibo.it
}

\author{
$4^{\text {th }}$ Luca Roffia \\ Advanced Research Center \\ On Electronic Systems \\ "Ercole De Castro" (ARCES) \\ Department of Electrical, \\ Electronic and Information \\ Engineering "Guglielmo Marconi" (DEI) \\ University of Bologna \\ Bologna, Italy \\ luca.roffia@unibo.it
}

\author{
$5^{\text {th }}$ Tullio Salmon Cinotti \\ Advanced Research Center \\ On Electronic Systems \\ "Ercole De Castro" (ARCES) \\ Department of Computer Science \\ and Engineering (DISI) \\ University of Bologna \\ Bologna, Italy \\ tullio.salmoncinotti@unibo.it
}

\begin{abstract}
This paper describes an application for the context aware tuning of the data rate of a battery powered LoRaWAN multi-sensor node equipped with sensors measuring soil features like water content, temperature, conductivity, moisture and water table depth. The application aims at saving as much power as possible, granting at the same time the detection and accurate profiling of events localized in time and space (e.g., due to sudden heavy rain). The tuning rules are based on the interplay between the context heterogeneous actors (sensor data, forecasts, current season, irrigation requests) mediated by a Linked Data distribution platform interconnected to multiple private and public networks. An interoperable application is provided, whose components can be easily extended and reused.

Index Terms-LoRaWAN, Interoperability, Linked Data, Context aware, Smart Sensor
\end{abstract}

\section{INTRODUCTION}

This paper is about the lesson learned and the actions taken during the development and the deployment of a platform for smart water management in agriculture [1] [2] [3]. The platform was developed within SWAMP [4], a project funded by the EU and by Brazil, and focused on the demonstration of Internet of Things (IoT) technologies in real-world scenarios.

Four pilots in the agricultural domain were proposed by SWAMP: one in Spain, one in Italy and two in Brazil. This paper is focused on the Italian pilot, located close to Reggio Emilia within the area managed by the Consorzio di Bonifica dell'Emilia Centrale (CBEC) [5]. CBEC manages the delivery of water to farmers through a network of open canals and the goal of the pilot is twofold: using sensors, weather forecast and models to detect crop water needs, and optimizing water delivery based on water requests made by the farmers (i.e., at the time of writing more than $15 \mathrm{~K}$ requests were filed during the ongoing irrigation season). Weather forecast and weather data are provided by ARPAE, the Italian Regional Environmental Protection Agency of the Emilia-Romagna region [6]. ARPAE also developed CRITERIA, the soil water balance model that provides water needs for some of the pilot farms and is integrated within the SWAMP platform [7] [8].

One of the main critical aspects in such a scenario is related to data collection and transmission. The lack of power sources and the extension of the area covered impose two main requirements: to use battery powered sensors and to have access to a low-power wide area network.

Both requirements were satisfied by adopting the LoRaWAN technology [9], supported by the PAIoT (Internet of Things for the Public Administration) network [10] implemented and maintained by LepidaScpa [11] and by designing a battery powered multi-sensor node. Saving power requires to limit the sampling rate on the sensor node as well as the data rate on the network. If on the one hand, the dynamics of the events in the reference domain (e.g., water table, soil moisture, soil temperature) are consistent with a low sampling rate, on the other hand the prompt detection and reaction to some kind 
of sporadic events (e.g., heavy rain) need a higher rate. This is the main focus of the paper: adapting the sampling and data rate of the LoraWAN multi-sensor nodes to the context detected by other system components.

\section{PAIOT NETWORK}

LepidaScpa is the in-house company of Emilia-Romagna in charge of providing broadband network to PAs (Public Administrations) in the region (Fig.1).

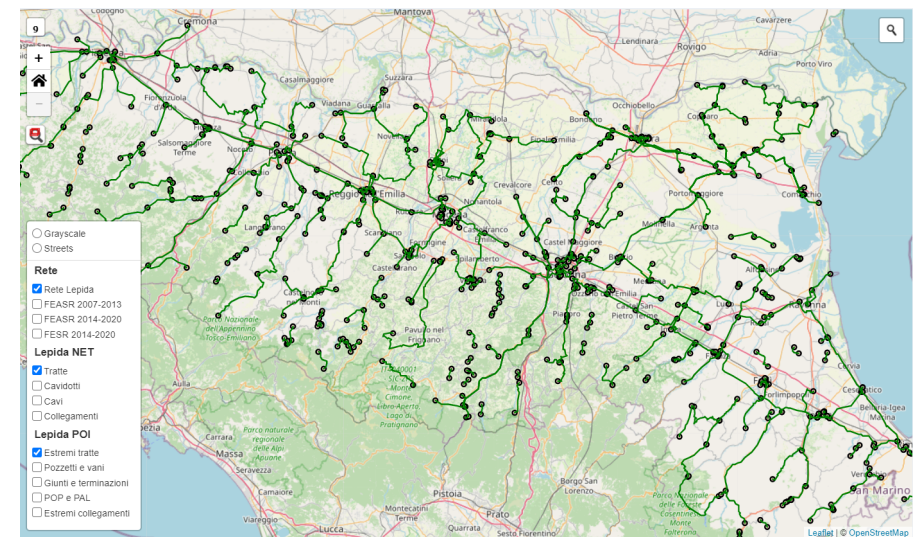

Fig. 1. Lepida broadband network

The simultaneous availability of Lo- $\mathrm{Ra}^{\mathrm{TM}}$ technology and Lepida broadband network are the right preconditions for the deployment of an IoT network at regional level, namely the PAIoT network, available to public administrations, companies and citizens. Fig. 2 shows the current status of implementation of the PAIoT network, which will cover all the provinces of the Emilia-Romagna region when fully operational.

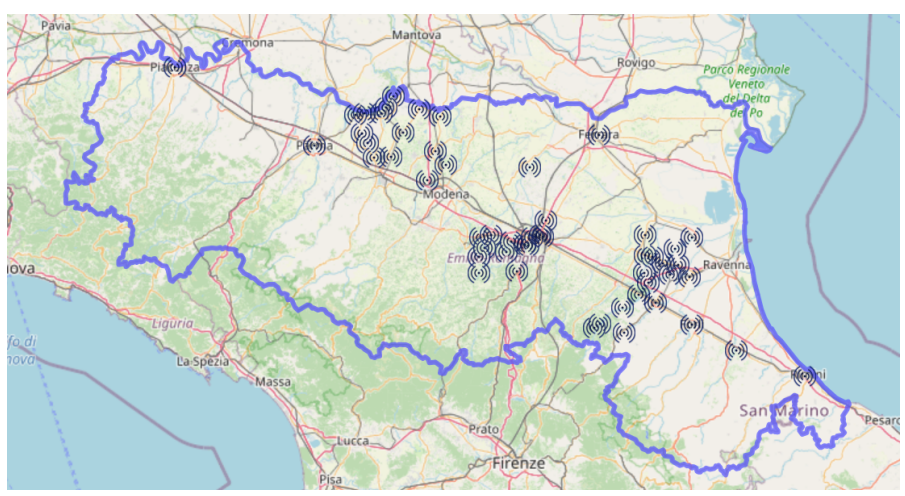

Fig. 2. retePaIoT network

The architecture of PAIoT network, based on Lepida broadband network, is described in Fig. 3 and relies on the following components:

- Sensors (of any kind) which send data to Lo-Ra ${ }^{\mathrm{TM}}$ gateways by means of LoRaWAN protocol (i.e., the multisensor node described in Section IV belongs to this category);

- LoRaWAN Gateways which receive data from LoRaWAN sensors are installed in any Access Point of Lepida

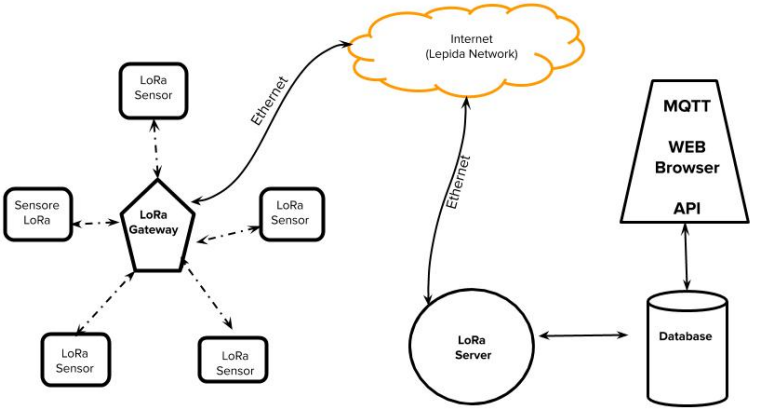

Fig. 3. PaIoT network architecture

network (PAL), in order to use Lepida broadband network to transmit data to Lo-Ra ${ }^{\mathrm{TM}}$ Server;

- Lo-Ra ${ }^{\mathrm{TM}}$ Server: virtual machine installed in one of the three regional data centers managed by LepidaScpa, that receives data from LoRaWAN Gateways through Lepida broadband network.

The data received from the LoRaWAN server are stored in a centralized database and are made available through different interfaces: MQTT protocol, API and web interfaces.

The initiative, implemented by LepidaScpa and funded by the Emilia-Romagna Region, has the following main objectives:

- Create a public IoT network where citizens, private companies and PA can integrate their own sensors, making them available to owners and PA entities.

- Allow citizens and private companies to collect data from their own sensors wherever they want to install them.

- Allow PA access to data collected by all the sensors installed in the territory for monitoring purposes.

- Enable IoT development through a unique Lo-RaTM network managed by PA with a rational usage of the frequencies and resources optimization.

- Map all the existing sensors in the territory through a sensor register providing all the technical parameters and the owner identification.

The availability of the PAIoT network at regional level is a facility for the installation of different types of sensors available to all the interested parties, public and private, reducing the implementation costs of monitoring systems only to those related to the purchase, installation and maintenance of sensors, and not those of the communication, collection and storage of the data. Furthermore, the PAIoT network uses the Lepida broadband network as a backbone for data collection, and benefits from its high level of reliability and security. Eventually, the PAIoT network coverage is extended at regional level, and it constitutes the prerequisite and opportunity for the replicability of pilot projects on a regional scale. 


\section{THE EFFECT OF THE SAMPLING RATE ON EVENTS DETECTION}

As stated above, saving power requires low sensor sampling rates and a low data rate on the network as well. If, on the one hand, the dynamics of the events in the reference domain (e.g., water table, soil moisture, soil temperature) are usually slow, on the other hand, the prompt detection and reaction to some kind of sporadic events (e.g., heavy rain) call for a higher sampling rate.

As an example taken from real data collected from a vineyard near Reggio Emilia (IT), Fig.4 and Fig.5 plot the water content in response to a heavy rain event (see Fig.6), and they compare data collected at 10 minutes and 1 hour sampling rate. The water content sensor is a Teros- 12 from Meter Group [12]. As expected, the same rain event shows different soil responses, depending on the sampling rate and the figures show how large these differences are. Particularly, the measures of the average water content increment rates per hour during the entire event are dramatically different $(59,8 \%$ versus $24,4 \%$ ), so that this measure at 60 minutes sampling rate should be considered meaningless.

Also the measure of the total contribution from the rain event to the soil water content is significantly affected by the sampling rate: the peak captured by the higher sampling rate (Fig.4) is lost when sampling hourly (Fig.5) (29,9\% and 24,4\% are the water content increase respectively measured at the considered sampling rates, as a response to a sudden and heavy - $40 \mathrm{~mm}$ - rainfall event).

As the battery life of the sensor nodes is affected by the sampling rate, this example motivates the need for variable sampling rates, if we want to properly measure the soil response to rain and irrigation events, without compromising battery life. The role of the algorithm presented in section $\mathrm{V}$ is to dynamically adjust the sensor sampling rates, based on contextual information provided by the platform.

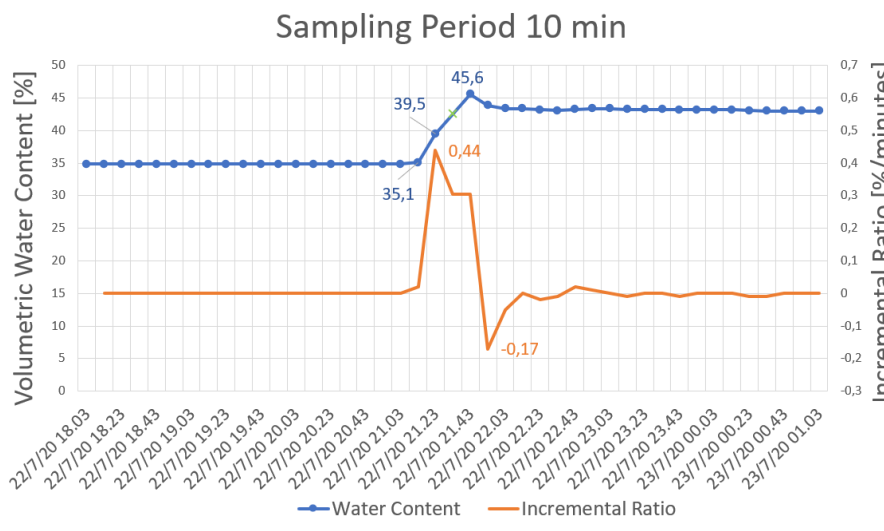

Fig. 4. Data collected with a sampling period of 10 minutes

\section{THE MULTI-SENSOR NODE}

The multi-sensor node is a hub-node for METER's sensors [12] designed by University of Bologna within the SWAMP

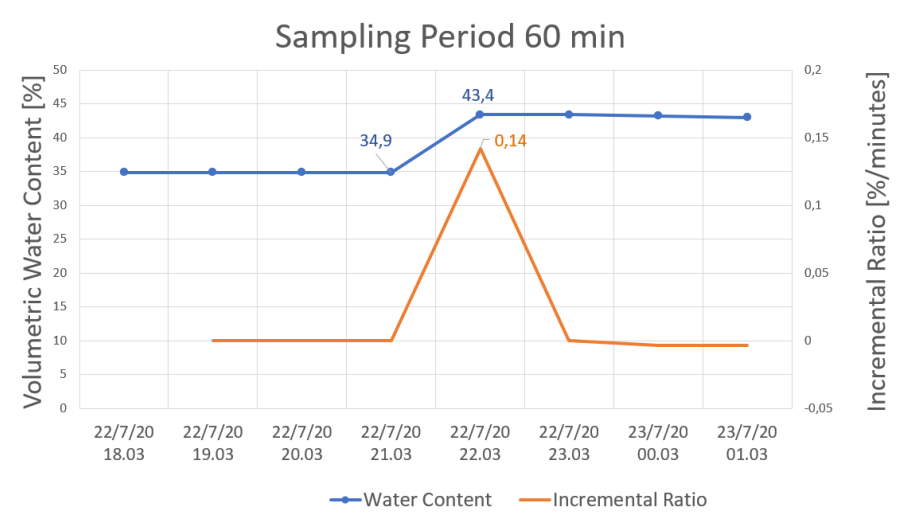

Fig. 5. Data collected with a sampling period of 1 hour

SWAMP - Smart Water Management Platform

Pluviometro Santa Maria - 003-R24H (Pioggia Cumulata Oggi)

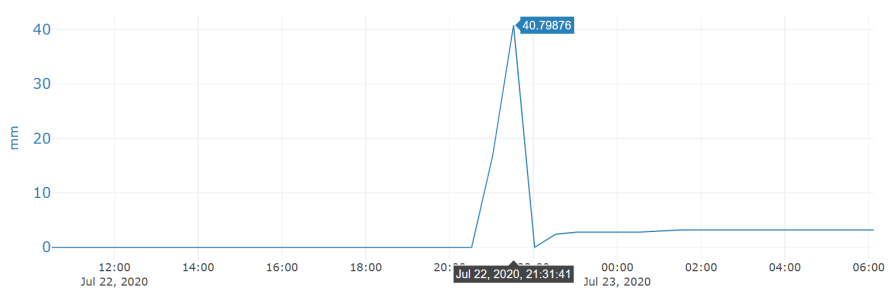

Fig. 6. Rain event shown by the SWAMP Water Distribution Application (data are provided by CBEC pluviometer)

project. The node (shown in Fig.7) can manage up to six general-purpose sensors such as soil moisture sensors, temperature sensors and water table sensors.

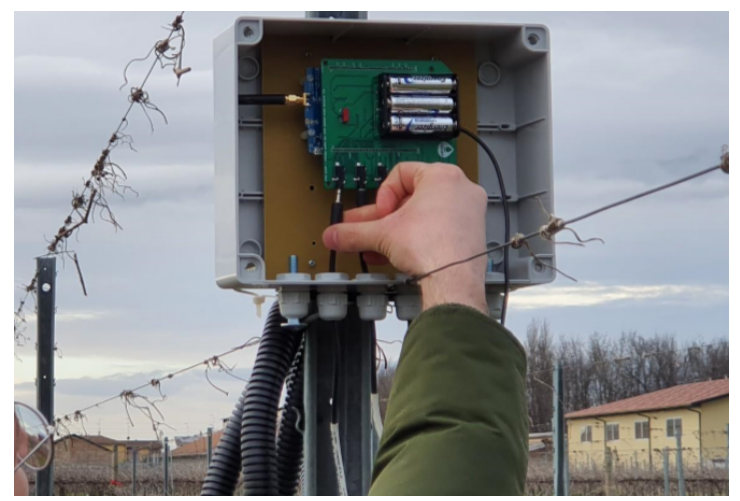

Fig. 7. A LoRaWAN low power multi-sensor node installed in the Italian pilot

The node has been designed for ultra-low power consumption and low cost, with production and maintenance in mind. In order to be compliant with these specifications, the node is powered by a STM32L0 microcontroller and the LoRa protocol is used for the communication between the server and the node. In particular, the node is a Class-A LoRaWAN node. In this configuration, the node collects and transmits sensor data to the server on fixed time intervals. During the 
rest of the time, the node is in low power sleep mode.

If on the one hand with fixed sampling and transmission intervals we limit the power consumption (i.e., the node does not need to stay awake in order to listen for commands from the server), on the other hand some events may need a faster reaction and may call for a temporarily higher sampling rate (e.g., a sudden summer storm or an abundant irrigation).

A solution to this problem is offered by LoRa downlink messages (from server to node), that can be used to change the sampling and transmission period at runtime. Because LoRaWAN Class-A devices can receive a downlink messages only after an uplink message (from node to server), some keepalive messages were added between two data messages. The period between two keep-alive messages and the number of keep-alive messages between two data messages can be reprogrammed at runtime through a downlink command messages.

The adaptive transmission period feature is useful when the duration of an event is different from the transmission period. An example is shown in Fig8. Assume that a keep-alive message is sent every 10 minutes and a data message is sent every 5 keep-alive messages. Therefore, the data message rate is 1 message/hour. If for example a new irrigation is scheduled in the evening, through a downlink command message, it is possible to set the number of keep-alive messages between two data messages to 1 . This will allow to have on the sever a new data value every 20 minutes and so a data rate of 3 messages/hour.

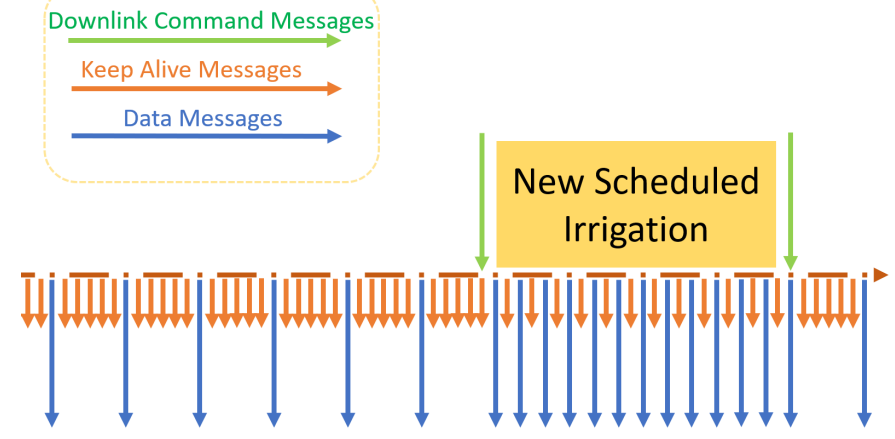

Fig. 8. Increasing the number of data messages per hour through downlink message to have a more detailed curve of the irrigation

The node also implements a feature to safe power when a low battery level is detected (Fig.9). In this case, for example, the keep-alive message period is set to 30 minutes and a data message is sent with the same delay after every keep-alive.

\section{CONTEXT AWARE TUNing OF THE MULTI-SENSOR NODE}

The SWAMP Italian pilot software architecture prvides several microservices which support web based applications like the Water Distribution Application (WDA) [13]. These microservices use an instance of the SPARQL Event Process Architecture (SEPA) [14] [15] as the main communication and storage facility. SEPA is a semantic data distribution system enabling the development of smart spaces and ambient

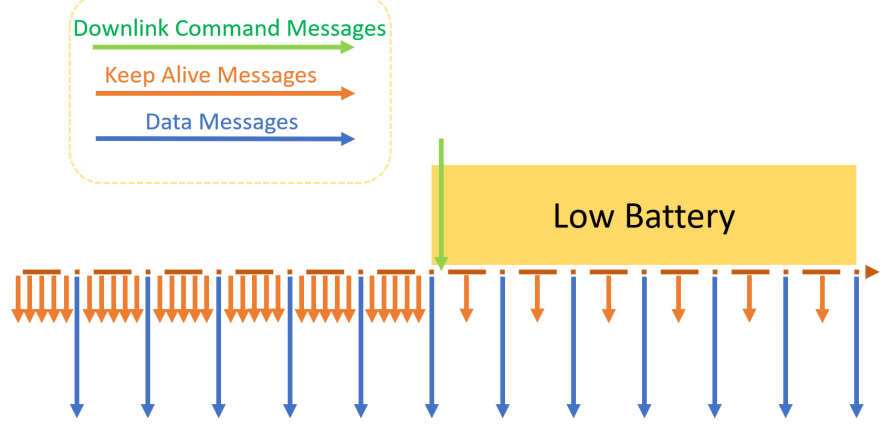

Fig. 9. Decreasing minutes beetwen two keep-alive messages in order to safe battery life

intelligence services [16]. In particular, the SEPA instance contains the knowledge base of the SWAMP cloud and it acts as a publish-subscribe broker who notifies subscribers about changes in its graph database. Leveraging, on this publishsubscribe pattern, other microservices can orchestrate each other to obtain the desired application logic.

In this context, we define a TuningSoilSensor microservice or smartlet that is in charge of setting the sampling time parameter of the multi-sensor node (see IV). This component takes into account contextual information to compute the correct wake-up time, such as weather forecast, battery level, sensor location, and scheduled irrigation plan. Focusing on this application, the architecture can be exemplified as shown in Fig. 10 .

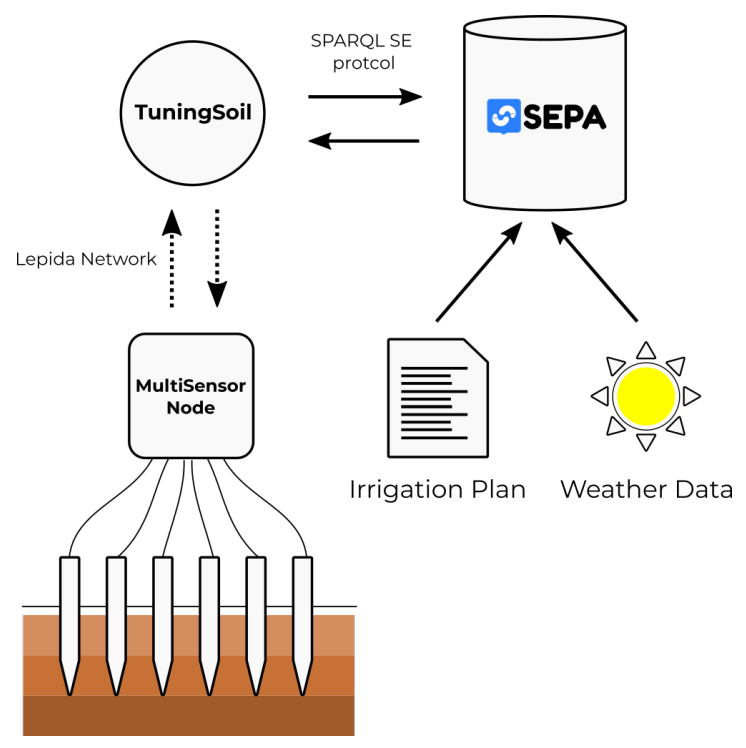

Fig. 10. TuningSoil software architecture

In practice, the TuningSoilSensor subscribes to the list of the active UNIBO Multisensor nodes using a SPARQL query [17]. Each sensor is described by a set of semantic tags which enable to retrieve its location and its battery level. When a new sensor is installed, the Smartlet is notified and it activates the sampling tuning watchdog. This watchdog is an internal 
service scheduled to run at each $D E L T A_{T}$. On each step, it queries the SEPA broker to obtain the sensor context and then executes the following algorithm:

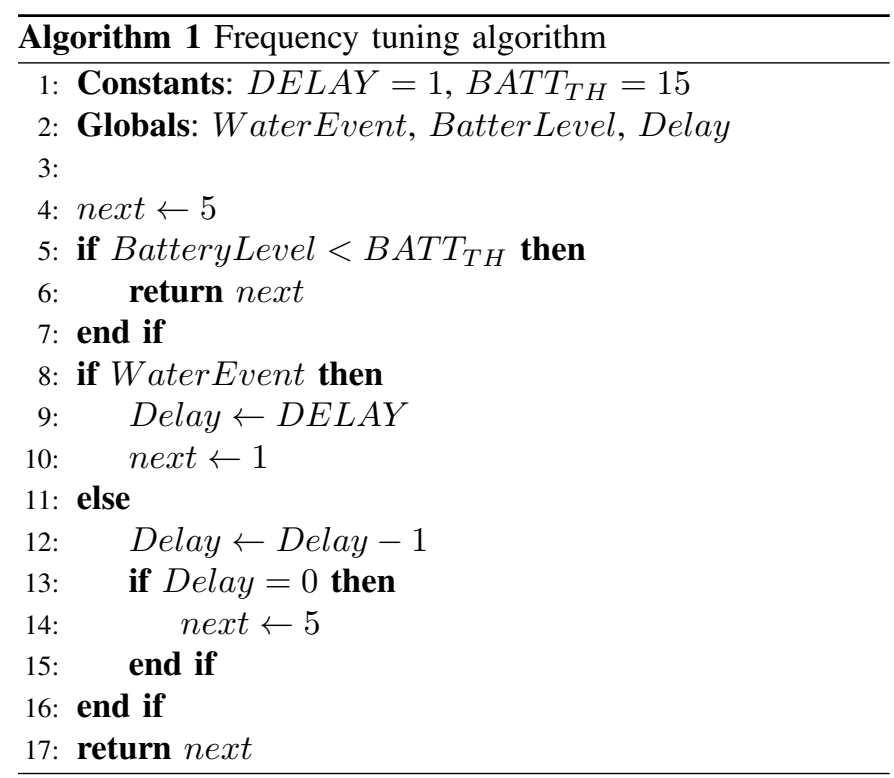

The algorithm takes as input a WaterEvent variable that is obtained by processing the weather forecast and the irrigation plan of the next hour. WaterEvent is true if in the next hour it will rain or if the farmer planned to irrigate, otherwise is false. Moreover, the watchdog requests the sensor battery level and initializes a $D E L A Y$ constant. This value defines the amount of DELT $A_{T}$ to wait until the end of the high sampling period. If we consider for example the soil water content, as a consequence of an irrigation it will react with a delay $X$ which is related to the soil characteristics and other variables. Therefore, in such a case, DELT $A_{T}$ allows to delay changing the sampling rate until the event of interest ended. Finally, the algorithm outputs the sampling frequency in terms of how many keep-alive messages should be sent before sending a data message. This output is then sent to the sensor using the downlink functionality of LoRaWAN. From this point on, the watchdog goes to sleep mode and the sensor node is reconfigured as soon as it wakes up again to send next keep-alive message.

\section{CONCLUSION}

The application presented in this paper is an example of how the agriculture domain can benefit from recent advances in technology. In particular, the advent of LoRaWAN opens up to new possibilities in terms of IoT applications. The effort made by LepidaScpa in providing an open LoRaWAN network to collect and store data from the field is a fundamental asset to reduce entry costs for stakeholders, both public and private, who want to deploy their own sensor network within the Emilia Romagna region. If on the one hand saving power is in line with green economy, on the other hand it is also a must when dealing with battery powered devices. At sensor level, power consumption can be reduced be reducing the sampling and transmission rate. But this can be in contrast with the sampling of higher frequency events. An automatic tuning of the sensor sampling rate can be performed based on the context where the sensor is operating in. With this respect, context detection and distribution is granted by SEPA which enables the development of distributed an context aware applications, including the one presented in this paper. Thanks to the Linked Data and Semantic Web technologies, SEPA provides an interoperability enabling technology which can be applied, with the same principles shown in this paper, also in several other IoT domains.

\section{ACKNOWLEDGMENT}

The work presented in this paper has been developed within the EU project SWAMP (Smart Water Management Platform), Project ID: 777112, Funded under: H2020EU.2.1.1.-INDUSTRIAL LEADERSHIP_Leadership in enabling and industrial technologies-Information and Communication Technologies (ICT).

\section{REFERENCES}

[1] C. Kamienski, J. Soininen, M. Taumberger, S. Fernandes, A. Toscano, T. S. Cinotti, R. F. Maia, and A. T. Neto, "Swamp: an iot-based smart water management platform for precision irrigation in agriculture," in 2018 Global Internet of Things Summit (GIoTS), pp. 1-6, 2018.

[2] I. Zyrianoff, A. Heideker, D. Silva, J. Kleinschmidt, J.-P. Soininen, T. Salmon Cinotti, and C. Kamienski, "Architecting and Deploying IoT Smart Applications: A Performance-Oriented Approach," Sensors, vol. 20 , no. 84,2020 .

[3] C. Kamienski, J. Kleinschmidt, J. Soininen, K. Kolehmainen, L. Roffia, M. Visoli, R. Filev Maia, and S. Fernandes, "Swamp: Smart water management platform overview and security challenges," in 2018 48th Annual IEEE/IFIP International Conference on Dependable Systems and Networks Workshops (DSN-W), pp. 49-50, 2018.

[4] "Swamp project web site." http://swamp-project.org/, 2020.

[5] "Consorzio di bonifica dell'emilia centrale." https://www.emiliacentrale.it/.

[6] "Arpae." https://www.arpae.it/.

[7] G. Villani et al., "Soil Water Balance Model CRITERIA-ID in SWAMP Project: Proof of Concept," 2018 23rd Conference of Open Innovations Association (FRUCT), 2018.

[8] “Criteria-1d." https://github.com/ARPA-SIMC/CRITERIA1D.

[9] “Lorawan.” https://lora-alliance.org/about-lorawan.

[10] "Rete pubblica iot." https://loragis.lepida.it/loragis/.

[11] "Lepida." https://www.lepida.net/en.

[12] "Meter group." https://www.metergroup.com/.

[13] "Water distribution application." http://mml.arces.unibo.it/swamp/wda/.

[14] L. Roffia, P. Azzoni, C. Aguzzi, F. Viola, F. Antoniazzi, and T. Salmon Cinotti, "Dynamic linked data: A sparql event processing architecture," Future Internet, vol. 10, no. 4, p. 36, 2018.

[15] L. Roffia, F. Morandi, J. Kiljander, A. D’Elia, F. Vergari, F. Viola, L. Bononi, and T. S. Cinotti, "A Semantic Publish-Subscribe Architecture for the Internet of Things," IEEE Internet of Things Journal, dec 2016.

[16] D. Korzun, E. Balandina, A. Kashevnik, S. Balandin, and F. Viola, eds., Ambient Intelligence Services in IoT Environments: Emerging Research and Opportunities. IGI Global, 2019.

[17] S. Harris and A. Seaborne, "Sparql 1.1 query language." https://www.w3.org/TR/sparql11-query/, 2013. 